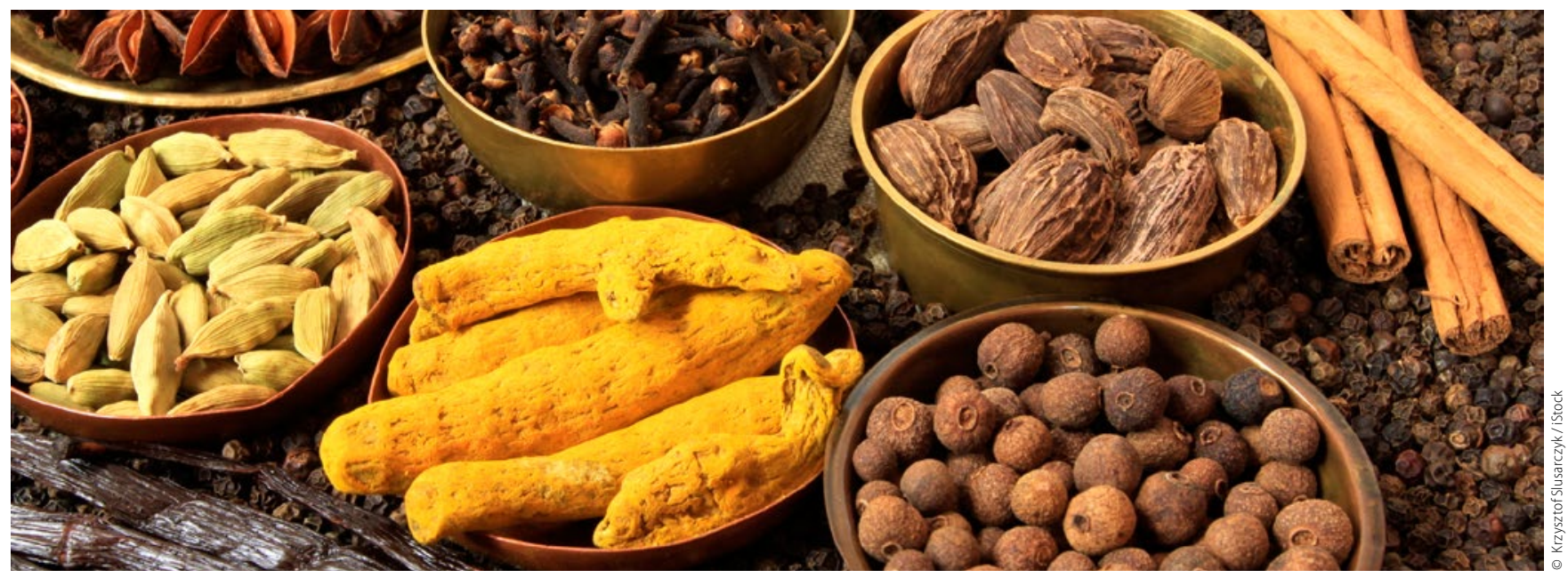

Ayurveda und moderne Zahnmedizin

\title{
Der Nutzen von Kurkuma für die Mundgesundheit
}

\begin{abstract}
Ayurveda gehört zu den ältesten medizinischen Lehren. Diese indische Heilkunst nutzt zum Erhalt der oralen Mundgesundheit verschiedene Kräuter, Kurkuma zum Beispiel. Das Gewürz wird auch gelber Ingwer, Safranwurzel oder Gelbwurzel genannt und ist eine der am häufigsten angewandten und auch wissenschaftlich untersuchten Heilpflanzen. Sie gehört zu den Ingwergewächsen und findet therapeutische Anwendung bei der Behandlung der Gingivitis, der

submukösen Fibrose oder der Tumorprävention.
\end{abstract}

Appliziert wird Kurkuma dabei systemisch per Mundspülung oder als topisch aufzutragende Gel-Zubereitung. Zur Behandlung der Gingivitis verglich eine prospektiv-randomisierte Studie eine zweiprozentige Chlorhexidin-Gelzubereitung mit einer Kurkuma-Gelzubereitung, indem beide Substanzen auf die Gingiva aufgetragen wurden. Die Ergebnisse hinsichtlich Plaqueund Gingivaindex unterschieden sich dabei nicht. Kurkuma war allerdings von den Studienteilnehmern geschmacklich als angenehmer empfunden worden, ohne zu Verfärbungen von Zähnen und Zunge zu führen (Kandwal et al. 2015). In der Zahnheilkunde soll Kurkuma außerdem auch zur Prävention der Karies dienlich sein.

Zur Behandlung der submukösen Fibrose, die zu den sogenannten präkanzerösen Konditionen gehört, zeigte Kurkuma wegen seiner antiinflammatorischen Wirkung gute Behandlungsergebnisse, indem es sowohl lokal als auch systemisch angewandt wurde. Im Rahmen dieser klinischen Studien konnten die Mundöffnung verbessert und die Schmerzen gelindert werden (Alok et al 2015). Zur Durchführung der Mundhygiene ver- trugen Patienten mit einer Radiochemotherapie-induzierten Mukositis laut einer weiteren klinischen, prospektiven Studie Kurkuma besser als eine CHX-Mundspüllösung (Patil et al. 2015). Im Hinblick auf die Tumorprävention könnte Kurkuma als Antioxidans von Bedeutung sein. Zur supportiven Tumortherapie beeinflusst es die Zellzyklusregulation und die Apoptose.

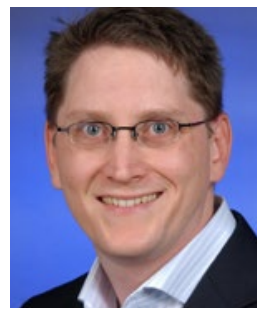

Prof. Dr. Dr. Felix P. Koch

Autor des Wissenschaftlichen Infodienstes, Lehrbeauftragter der Uniklinik Frankfurt, Praxisklinik für Mund-, Kiefer- und plastische Gesichtschirurgie in Wiesbaden. Mail: wid@fvdz.de 